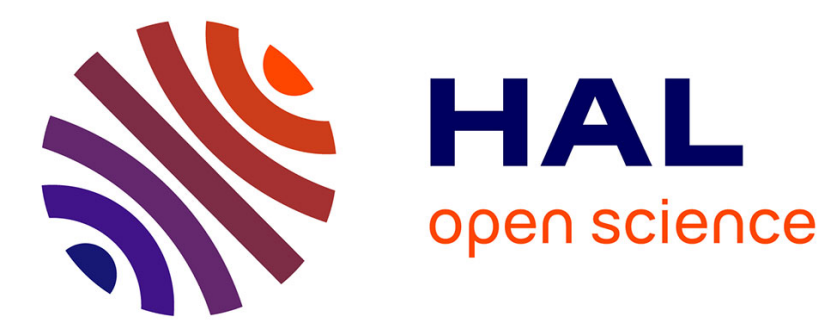

\title{
Energy release rate of small cracks in hyperelastic materials
}

\author{
Malik Aït-Bachir, W. V. Mars, Erwan Verron
}

\section{To cite this version:}

Malik Aït-Bachir, W. V. Mars, Erwan Verron. Energy release rate of small cracks in hyperelastic materials. International Journal of Non-Linear Mechanics, 2012, 47 (4), pp.22-29. 10.1016/j.ijnonlinmec.2012.03.001 . hal-01006798

\section{HAL Id: hal-01006798 \\ https://hal.science/hal-01006798}

Submitted on 16 Jun 2014

HAL is a multi-disciplinary open access archive for the deposit and dissemination of scientific research documents, whether they are published or not. The documents may come from teaching and research institutions in France or abroad, or from public or private research centers.
L'archive ouverte pluridisciplinaire HAL, est destinée au dépôt et à la diffusion de documents scientifiques de niveau recherche, publiés ou non, émanant des établissements d'enseignement et de recherche français ou étrangers, des laboratoires publics ou privés. 


\title{
Energy release rate of small cracks in hyperelastic materials
}

\author{
M. Aït-Bachir ${ }^{\text {a }}$, W.V. Mars ${ }^{\mathrm{b}}$, E. Verron ${ }^{\mathrm{a}}$ \\ a LUNAM Université, Ecole Centrale de Nantes, GeM, UMR CNRS 6183, BP 92101, 44321 Nantes cedex 3, France \\ ${ }^{\mathrm{b}}$ Cooper Tire and Rubber Company, 701 Lima Avenue, Findlay, Ohio 45840, USA
}

\begin{abstract}
The energy release rate of a small crack in an infinite hyperelastic medium, and subjected to large strain multiaxial loading conditions, is derived by considering the balance of configurational stresses acting on two planes: one cutting the center of the crack face, and the other at an infinite distance in front of the crack tip. The analysis establishes that the energy release rate of a small crack is always proportional to the size of the crack, irrespective of the loading conditions and the crack orientation. The balance of configurational stresses is illustrated for several benchmark cases including simple extension, pure shear and equibiaxial extension, and for perpendicular and inclined cracks.
\end{abstract}

\author{
Keywords: \\ Cracks \\ Energy release rate \\ Configurational stress \\ Hyperelasticity \\ Rubber-like materials
}

\section{Introduction}

Under arbitrary loading conditions, a microscopic inhomogeneity existing in a material experiences forces that tend to drive its growth. One well-known and classical approach to evaluate these forces is to idealize the inhomogeneity as a crack, and to calculate the energy release rate defined in the general framework of Fracture Mechanics [1,2]. Such an application may, for example, be made via finite element analysis in which the crack is considered as part of the meshed geometry. However, because small cracks may take place at any point and in any orientation, placing a crack at the most critical location and with the most critical orientation requires information unavailable at the outset of such an analysis. In this study, we are seeking an alternative approach that addresses this problem directly: a continuum theory capable to provide, for every material point in a body, the driving force acting on a hypothetical idealized small crack. More specifically, we would like to compute the energy release rate of an idealized crack per unit of crack length (so that this parameter has unit of energy per unit of volume and quantifies the energy density available at each point for driving crack growth) in terms of stress and strain at each material point, and of the given orientation of the idealized crack.

In the special context of fatigue of soft materials, several workers have already discussed or proposed heuristically derived inexact solutions of the type sought. For example, Roach [3] conducted various experiments to compare crack response in simple tension and equibiaxial tension, emphasizing that only a part of the strain energy density is available for driving small crack growth. In order to address crack nucleation problems, Mars and Fatemi [4] derived the Cracking Energy Density, a continuum parameter aimed at a more general-purpose accounting of available strain energy density for the growth of a crack in a given material plane. Recently, several authors have also proposed novel continuum schemes, again with the aim of strengthening predictive capabilities for fatigue crack nucleation in rubber materials [5-9]. To date, none of these quantities has enjoyed a fully rigorous connection to a Fracture Mechanics solution, but they have provided valuable insight and motivation towards the more complete solution, and a means to move forward with engineering analysis tasks.

We have noticed the recent emphasize led on the Configurational Mechanics theory, which seems well-adapted to our purpose. The interested reader can consult the works of Maugin for a detailed and motivating review $[10,11]$. Suffice it here to say that Configurational Mechanics is a restatement of the basic physical conservation and balance laws: as classically stated, these laws are written in terms of spatially measured variables, i.e. stresses and physical forces; however, in Configurational Mechanics, the laws are restated in terms of variables measured in the material space, i.e. the abstract set of particles that make up the body [12]. In this framework, basic quantities such as the displacement and the force take on new meanings. For example, displacement of a crack tip in the material space represents crack growth. Likewise, the force that acts at this point in the material space represents the energy release rate of the crack. Although the general theory is new, it 
encompasses the classical theories of Fracture Mechanics and it has already started to find successful application to hyperelastic fracture mechanics problems: Steinmann et al. [13] has shown that finite element calculation procedures for the energy release rate can be greatly simplified through this framework, Verron [14] has shown that the framework provides a simple and new way to understand the mechanics of the trousers tear specimen, and Kaliske and coworkers [15] have used the framework to compute the energy release rates in tires and rubber parts. The reader can refer to [16] for a review of the use of Configurational Mechanics for rubber problems.

Our present objective is to use the tools of Configurational Mechanics to derive the energy release rate of a center-cracked region under arbitrary far-field homogeneous multiaxial loading conditions; for the sake of simplicity only the 2D problem is investigated. We idealize the inhomogeneity as a straight through-crack and only consider the case of steady-state crack growth. We will show that, in general, the energy release rate can be understood in terms of a balance between configurational stresses that are uniform at the far-field and vary in the near-field due to the presence of the crack. It is seen that the near-field variations of configurational stress are associated with unloading of crack faces.

\section{Energy release rate of a small crack}

The analysis is targeted to the specific case of a crack with known ideal shape (straight-line through-crack in 2D), and with suitably restricted dimensions. In the following sections, we define the parameters considered here, and describe the restrictions on crack geometry that we will adopt. Then, the energy release rate is derived.

\subsection{Definition of the energy release rate}

In a deformed hyperelastic body containing a crack, the total strain energy $U$ in the body is a function of the geometry of the body, of the loading conditions and also of the crack characteristics. For two-dimensional problems, the crack is a line of length $A$ (in the undeformed state) which is a part of the boundary of the body (external or internal boundary). The crack line is bounded by a point-the crack tip-which experiences mechanical fields capable of rupturing the material. When this happens, the crack tip advances through the material, and the undeformed length of the crack increases. The tendency of the crack to propagate is characterized by the change of energy induced by crack growth, via the energy release rate $T$ defined as follows:

$T=-\frac{\partial(U-V)}{\partial A}$,

where $V$ is the work of external forces. Under conditions of constant boundary displacement, external forces do not work and $T$ reduces to

$T=-\left.\frac{\partial U}{\partial A}\right|_{\text {disp }}$,

in which the subscript disp means that the boundary displacement is constant.

Rivlin and Thomas were the first to apply this quantity to the analysis of cracks in rubber [2]. They showed experimentally that the energy release rate uniquely characterizes the loading state of the crack tip and is independent of the particular geometry of both the specimen and the crack. Because of this, the energy release rate is a fundamental and convenient criterion for describing the conditions that cause crack growth.
Different methods for the computation of energy release rates have been developed, e.g. the stress and displacement correlation method, the crack closure integrals, the nodal release method, etc. [17-21]. Nevertheless, an important early method for computing the energy release rate consists in using the Rice's J-integral [22]. For two-dimensional problems, the $J$-integral is a contour integral that is evaluated on a path $\Gamma$ that starts on one of the crack faces and ends on the other

$J=\lim _{\Gamma \rightarrow 0} \int_{\Gamma}\left(w n_{1}-\mathbf{n} \boldsymbol{\sigma} \frac{\partial \mathbf{u}}{\partial x_{1}}\right) d \Gamma$,

for small strain problems. In this equation, $w$ stands for the strain energy density, $\mathbf{n}$ is the outward normal vector to the contour, $n_{1}$ is its component in the crack direction $\left(x_{1}\right), \boldsymbol{\sigma}$ is the Cauchy stress tensor and finally $\mathbf{u}$ is the displacement vector. In the special case of elasticity, the integral is revealed path-independent and the limit operator can be omitted. The previous result can be extended to large strain; the corresponding integral can then be defined in the reference configuration and is expressed as [10]

$J=\mathbf{q} \cdot \int_{\Gamma} \mathbf{\Sigma} \mathbf{N} d \Gamma$,

where $\mathbf{q}$ is the crack direction, $\mathbf{N}$ is the outward normal to the path $\Gamma$ and $\boldsymbol{\Sigma}$ is the configurational stress tensor defined as [23-25]

$\boldsymbol{\Sigma}=W \mathbf{I}-\mathbf{F}^{T} \mathbf{P}=W \mathbf{I}-\mathbf{C S}$

In this equation, $W$ is the strain energy density per unit of undeformed volume, $\mathbf{I}$ is the identity tensor, $\mathbf{F}$ is the deformation gradient, $\mathbf{C}$ is the right Cauchy-Green strain tensor, and $\mathbf{P}$ and $\mathbf{S}$ are respectively the first and second Piola-Kirchhoff stress tensors. Note that $\Sigma$ depends on the material response through the constitutive equation which relates $\mathbf{S}, \mathbf{C}$ and $W$.

Remark 1. Here it is assumed that a crack will grow in the direction aligned with its direction: a straight-line through-crack will always grow in its existing direction.

\subsection{Definition of a small crack}

The material is considered hyperelastic, isotropic, incompressible and homogeneous. We consider cracks which are often many orders of magnitude smaller than the geometric features of the body under analysis, and we idealize them as straight through-cracks. It is also assumed that at the scale of the Representative Volume Element, the scale of Continuum Mechanics, these idealized cracks (i) exist in every possible orientation with the same probability, (ii) their density is sufficiently small to consider that they do not interact with each other, (iii) they develop each at its own rate. Consequently, we only study a single linear through-crack embedded in an infinite region of homogeneously strained material. Moreover, we consider that this crack ever remains a straight through-crack and grows in its length direction and that the far-field strain and stress considered match to the strain and stress at the point under analysis in the macroscopic non-homogeneously strained body. Finally, we restrict our attention to steady-state crack propagation and consider that the configuration of the damage zone, i.e. in terms of the near-fields, does not change during its relative motion induced by crack growth. More precisely, it means that (i) we do not consider the early start of crack growth during which the crack propagates and the damage zone forms simultaneously, but the steady state for which the damage zone has reached its equilibrium stage, and that (ii) the damage zone does not reach the boundary of the domain because its configuration would become complicated. Indeed, the latter requirement can be 
seen as a part of the definition of a small crack, because our theory will apply only if a sufficiently large region (in which the far-fields can be considered uniform) surrounds the crack.

\subsection{Derivation of the small crack energy release rate}

As suggested in Fig. 1, we define a coordinates system in which the crack direction remains fixed. The problem consists in deriving the energy release rate of the crack, and to relate the energy release rate per unit of crack length to the homogeneous strain taking place in regions far from the crack.

As stated above, the energy release rate of the crack can be evaluated through the $J$-integral calculated on a path surrounding the crack tip. In order to isolate the variations of a specific component of the configurational stress tensor in the expression of the energy release rate and to emphasize in a very simple manner how the competition between the near-field and the farfield quantities makes up for the energy release rate of the small crack, it is a judicious choice to consider the contour shown in Fig. 1 wherein the small crack is aligned with $\mathbf{e}_{1}$ (shown in the undeformed configuration). More precisely, this contour, denoted $\Gamma$, traces out a rectangular region with arbitrarily height $2 R$ and width $D$, starts at the center of the top crack face and follows through the segments labeled $\Gamma_{+}, \Gamma_{A}, \Gamma_{B}, \Gamma_{C}$, and $\Gamma_{-}$, and ends at the center of the bottom crack face. Thus, the $J$-integral is written as the summation of individual contributions of each segment

$J=\int_{\Gamma_{+} \cup \Gamma_{A} \cup \Gamma_{B} \cup \Gamma_{C} \cup \Gamma_{-}} \mathbf{e}_{1} \cdot \mathbf{\Sigma} \mathbf{N} d \Gamma$.

At this step of the calculation, there is no distinction between a small crack and an ordinary crack: Eq. (6) applies whatever the size of the crack. However a small crack is by definition embedded in an infinite medium where far from the crack the mechanical fields are uniform and equal to those in a homogeneous material subjected to the same state of stress and strain at its boundary. Thus under the small crack assumption, infinite values are considered for $R / c$ and $D / c$. The segments $\Gamma_{A}, \Gamma_{B}$ and $\Gamma_{C}$ then lie in the far-field region wherein the configurational stress tensor is uniform and denoted $\Sigma^{\infty}$.

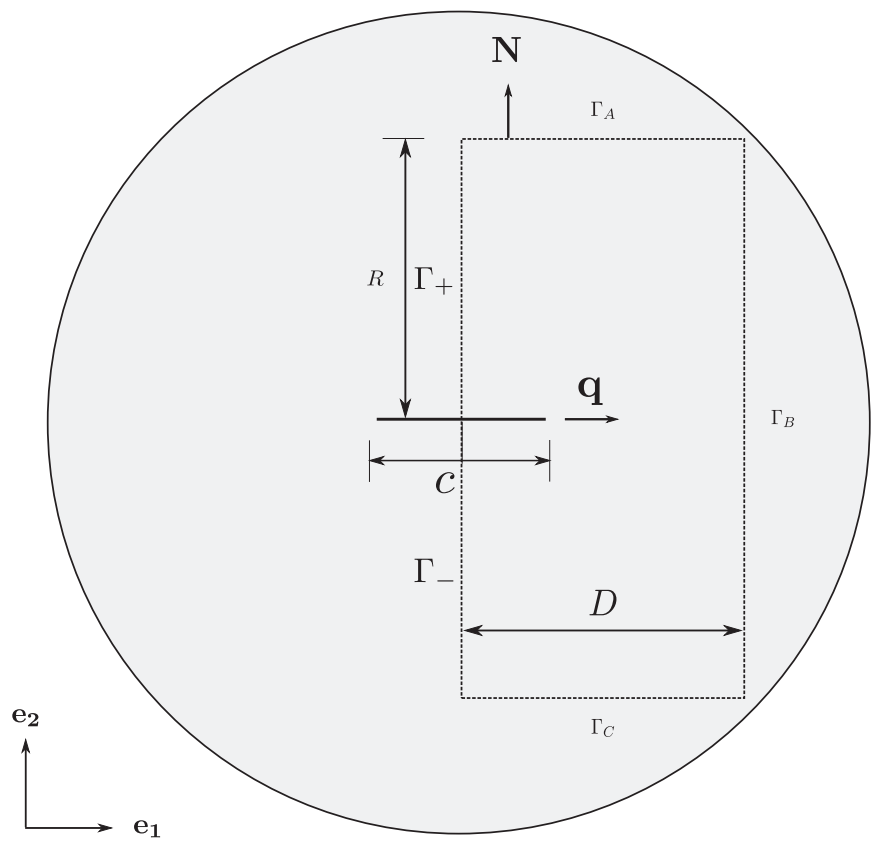

Fig. 1. Coordinates system and contour for the evaluation of the $J$-integral
Let us denote $\Sigma_{11}(l)$ the 11-component of the configurational stress tensor along the path $\Gamma_{-} \cup \Gamma_{+}$at an algebraic distance $l$ from the center of the crack. Then, the different contributions to the $J$-integral are given by

- Segment $\Gamma_{+}: \mathbf{N}=-\mathbf{e}_{1}$

$$
\int_{\Gamma_{+}} \mathbf{e}_{1} \cdot \mathbf{\Sigma} \mathbf{N} d \Gamma=-\int_{0}^{R} \Sigma_{11}(l) d l,
$$

- Segment $\Gamma_{A}: \mathbf{N}=\mathbf{e}_{2}$

$$
\int_{\Gamma_{A}} \mathbf{e}_{1} \cdot \mathbf{\Sigma} \mathbf{N} d \Gamma=\Sigma_{12}^{\infty} D
$$

- Segment $\Gamma_{B}: \mathbf{N}=\mathbf{e}_{1}$

$$
\int_{\Gamma_{B}} \mathbf{e}_{1} \cdot \mathbf{\Sigma} \mathbf{N} d \Gamma=2 \Sigma_{11}^{\infty} R
$$

- Segment $\Gamma_{C}: \mathbf{N}=-\mathbf{e}_{2}$

$$
\int_{\Gamma_{C}} \mathbf{e}_{1} \cdot \mathbf{\Sigma} \mathbf{N} d \Gamma=-\Sigma_{12}^{\infty} D
$$

- Segment $\Gamma_{-}: \mathbf{N}=-\mathbf{e}_{1}$

$$
\int_{\Gamma_{-}} \mathbf{e}_{1} \cdot \mathbf{\Sigma} \mathbf{N} d \Gamma=-\int_{-R}^{0} \Sigma_{11}(l) d l
$$

By summing all these contributions, we obtain the following expression of the energy release rate of a small crack

$J=\lim _{R \rightarrow+\infty} \int_{-R}^{R}\left(\Sigma_{11}^{\infty}-\Sigma_{11}(l)\right) d l$.

Invoking now the central symmetry with respect to the crack center of both the geometry and the boundary loading conditions, this expression of $J$ can be simplified. Consider the material point $M$ located at $\mathbf{X}_{M}$ in the reference configuration that moves to $\mathbf{X}_{M}$ in the deformed configuration, then its symmetric counterpart $M^{\prime}$ located at $\mathbf{X}_{M^{\prime}}=-\mathbf{X}_{M}$ moves to $\mathbf{x}_{M^{\prime}}=-\mathbf{X}_{M}$. Thus, the deformation gradient $\mathbf{F}=\partial \mathbf{X} / \partial \mathbf{X}$ is identical in $M$ and $M^{\prime}$; consequently, all strain and stress quantities are identical for these two particles. Thus, the previous expression of $J$ reduces to

$J=2 \int_{0}^{+\infty}\left(\Sigma_{11}^{\infty}-\Sigma_{11}(l)\right) d l$.

Remark 2. No problem occurs at infinity in the sense that $\Sigma_{11}(l)$ tends to $\Sigma_{11}^{\infty}$ as $l \rightarrow \infty$ and thus the integrand $\left(\Sigma_{11}^{\infty}-\Sigma_{11}(l)\right)$ vanishes as $l \rightarrow \infty$.

Eq. (13) reveals that the energy release rate of a small crack under arbitrary multiaxial loading conditions corresponds to the imbalance between the uniform far-field configurational stress acting on the right-hand side boundary of the integration path $\Gamma_{B}$ and the varying near-field configurational stress acting on its lefthand side boundary $\Gamma_{+}$. Indeed the right-hand side boundary is loaded by the constant value $\Sigma_{11}^{\infty}$, whereas the left-hand side boundary admits an equal and opposite asymptote to the value $\Sigma_{11}^{\infty}$ at an infinite distance from the crack, but is unbalanced near the crack due to the effects of crack face unloading. This imbalance of the configurational stresses between the left and right contributions of the path-integral reveals the physical origin of the energy release rate. Note that the relationship between 
unloading of crack faces and the energy release rate of small cracks under multiaxial loading conditions has been previously highlighted $[3,4]$.

\subsection{A further result: proportionality of J with respect to the crack length}

We recall that since the study of Rivlin and Thomas [2] based on dimensional analysis, it is widely recognized that under uniaxial tension the energy release rate of a small crack perpendicular to the tensile direction is proportional to its length. More recently, Yeoh extended this result to the cases of equibiaxial tension and pure shear but still for a small crack aligned with the principal stretch directions [26]. On intuitively assuming that this proportionality relationship remains valid under any arbitrary multiaxial loading conditions, Mars [4] developed his predictor which has enjoyed some empirical success for rubber fatigue. We point out here that although his intuition turns out to be right in practice, it has never been rigorously demonstrated.

The objective of the present section is to make use of the expression of the energy release of a small crack Eq. (13) and of a simple dimensional analysis to demonstrate the proportionality of the energy release rate of a small crack with respect to the crack length under any arbitrary multiaxial loading conditions and thus definitely prove what all the above-mentioned authors have postulated.

Let us consider two different small cracks: one with length $c$ and the other one with length $k c$ where $k$ is a strictly positive real number. Both are subjected to the same far-field conditions. Using Eq. (13), the energy release rate of the crack of length $c$ is

$J(c)=2 \int_{0}^{+\infty}\left(\Sigma_{11}^{\infty}-\Sigma_{11}^{c}(l)\right) d l$,

while the energy release rate of the crack of length $k c$ is

$J(k c)=2 \int_{0}^{+\infty}\left(\Sigma_{11}^{\infty}-\Sigma_{11}^{k c}(l)\right) d l$,

where $\Sigma_{11}^{c}$ (respectively $\Sigma_{11}^{k c}$ ) denotes the configurational stress associated with the crack of length $c$ (respectively of length $k c$ ). By using the substitution $l=k l^{\prime}$ and $d l=k d l^{\prime}$, the latter integral becomes

$J(k c)=2 k \int_{0}^{+\infty}\left(\Sigma_{11}^{\infty}-\Sigma_{11}^{k c}\left(k l^{\prime}\right)\right) d l^{\prime}$.

Let us focus now on the effect of multiplying the crack length by a factor $k$ on the transformation of the mechanical fields with respect to the original problem of a crack of length $c$. In this way, we consider a hyperelastic isotropic domain $\mathcal{D}^{c}$ surrounding the crack of length $c$. The dimensions of the domain are sufficiently large such that the crack is a small crack in the sense of our previous definition in Section 2.2. Similarly, we introduce the proportional replica of $\mathcal{D}^{c}$ by the scale factor $k$; it is denoted $\mathcal{D}^{k c}$. Note that the present derivation is valid if and only if the crack of length $k c$ is also a small crack, i.e. if the domain $\mathcal{D}^{k c}$ is small as compared to the size of the Representative Volume Element. If this assumption is satisfied, the two domains admit the same boundary conditions: normal tractions $\boldsymbol{\sigma n}$ at crack faces vanish and tractions at the external boundary are equal to the far-field tractions. Moreover, as the material is elastic the stress far-field is unique and simply related to the strain far-field by the constitutive equation. So, the boundary conditions being identical, solving the equilibrium equation will lead to identical solutions: denoting $M^{c}$ an arbitrary particle of $\mathcal{D}^{c}$ which position is $\mathbf{X}^{c}$, and $M^{k c}$ its geometrical counterpart in $\mathcal{D}^{k c}$ which position is $k \mathbf{X}^{c}$, we have

$\boldsymbol{\sigma}^{k c}\left(M^{k c}\right)=\boldsymbol{\sigma}^{c}\left(M^{c}\right)$, where $\boldsymbol{\sigma}^{c}$ and $\boldsymbol{\sigma}^{k c}$ represent the Cauchy stress field in $\mathcal{D}^{c}$ and $\mathcal{D}^{k c}$, respectively. Obviously this result extends to all the tensorial fields, i.e. strain tensors and other stress tensors, and particularly to the configurational stress tensor. Thus, particularizing this result to the 11-component of the configurational stress tensor on the segment $\Gamma^{+}$leads to

$\Sigma_{11}^{k c}(k l)=\Sigma_{11}^{c}(l)$ on $\Gamma_{+}$.

Then, substituting Eq. (18) into Eq. (16) and recalling Eq. (14) give the proportionality relationship

$J(k c)=k J(c)$.

So, we have demonstrated that for a fixed far-field state of strain and stress, the energy release rate of a small crack in a hyperelastic isotropic material varies proportionally with the crack length. Note that this result is valid under any arbitrary multiaxial loading conditions; in other words, even when the small crack is not aligned with the principal stretch directions which was a priori not obvious.

Moreover, Eq. (19) signifies that it is sufficient to know the value of the $J$-integral for one given crack length to calculate $J$ for all crack lengths: assuming that the value of $J$ is known for a given crack of length $c_{0}$, the value of $J$ for another crack length $c$ is simply given by

$J(c)=\frac{J\left(c_{0}\right)}{c_{0}} c$.

Eq. (20) proves that the energy release rate of a small crack embedded in a hyperelastic isotropic material and subjected to arbitrary multiaxial loading is always proportional to the crack length. Indeed, Eq. (19) means that for a fixed far-field state of strain and stress the factor $J(c) / c$ is strictly independent from the crack length. This remark leads us to conclude that $J(c) / c$ only depends on the far-field properties to which the small crack is subjected. Denoting $P$ this quantity, it can be summarized by

$\frac{J(c)}{c}=P($ far-field $)$.

\section{Results and discussion}

In this section, we first validate the previous derivation, i.e. the expression $J$ in terms of the configurational stress Eq. (13) and its proportionality with respect to the crack length Eq. (19), and second we analyze more specifically the near-field $\Sigma_{11}(l)$ in order to investigate the relationship Eq. (21).

In the following, we consider a domain that contains an inclined small crack of length $c$ as shown in Fig. 2. The plane stress assumption is adopted; thus the incompressibility of the

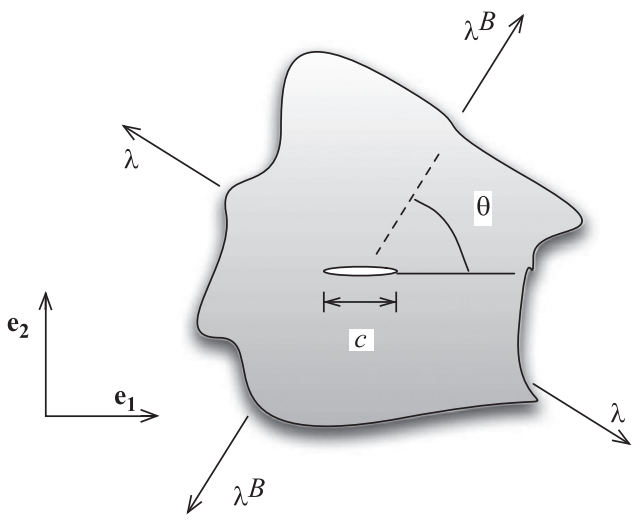

Fig. 2. Domain with an inclined center crack. 
material is simply handled. The far-field multiaxial straining is imposed through the set of parameters $(\lambda, B, \theta)$ where:

- $\lambda$ is the maximum principal stretch ratio in the plane $\left(\mathbf{e}_{1}, \mathbf{e}_{2}\right)$

- $\lambda^{B}$ is the second principal stretch ratio in the plane $\left(\mathbf{e}_{1}, \mathbf{e}_{2}\right)$,

- and $\theta$ is the crack orientation with respect to the second principal stretch direction.

The 2D method developed in [27] is used: this simple method easily accounts for the case in which the crack is not aligned with the principal stretch directions $\left(\theta \neq 0^{\circ}\right.$ and $\left.\theta \neq 90^{\circ}\right)$ which makes it ideally suited for finite element analysis of the response of a small crack near-field and for calculation of the energy release rate.

The finite element software Abaqus (version 6.8) was used for all numerical computations. The features of our finite element model can be summarized as follow.

- Constitutive equation. The theoretical conclusions drawn above are not based on the hyperelastic constitutive equation considered, for the sake of simplicity the incompressible neo-Hookean constitutive equation is chosen to model the behavior of the rubber-like material,

$W=C\left(I_{1}-3\right)$

where $I_{1}$ is the first invariant of the right Cauchy-Green strain tensor, and $C$ is the unique material parameter set to $1 \mathrm{MPa}$.

- Domain. For convenience, the far-field boundary is considered as a circular region surrounding the crack. In order to comply with the small crack assumption, a scale factor equal to 40 between the diameter of the far-field circular geometry and the crack length has been chosen. The crack size is set to $c=10 \mathrm{~mm}$.

- Loading conditions. The homogeneous multiaxial strain farfield is prescribed through a DISP subroutine that consists calculating the displacement of boundary nodes that leads to the chosen strain far-field (see [27] for details).

- Modeling the crack. The straight through-crack is modeled by a "seam" which is an Abaqus tool that permits to place overlapping duplicate nodes along a face in a model that is originally closed but can open during an analysis.

- Finite elements and mesh. 4-node linear 2D shell element (CSP4) finite elements are employed. The model is meshed and the mesh density is increased near the crack. The final mesh contains 32988 elements and 33093 nodes.

- Computation of J. The configurational stress tensor was evaluated by implementing Eq. (5) in Abaqus via the UVARM user-defined subroutine. Moreover, because of the necessarily finite dimension of the model, we have to settle for the evaluation of improper integral on an unbounded domain (Eq. (13)) with the help of an integral on the bounded domain $\Gamma_{+}$that starts from the center of the crack and ends on the far-field boundary. Note that $\Sigma_{11}^{\infty}$ is approximated here by the value of $\Sigma_{11}(l)$ at the intersection of $\Gamma_{+}$with the far-field boundary. The integral of $\Sigma_{11}^{\infty}-\Sigma_{11}(l)$ is calculated by the trapeze method where the two trapeze vertices correspond to two successive nodes along the path $\Gamma_{+}$at which the value of $\Sigma_{11}$ is computed.

\subsection{Validation of the derivation}

\subsubsection{The imbalance of configurational stress yields to the energy} release rate

Our first objective is to verify that the right-hand side term of Eq. (13) is equal to the energy release rate of the problem considered. Given that Abaqus has a tool that permits the direct evaluation of the $J$-integral, we compute the energy release rate with this feature on the one hand and the area enclosed in a plot of the integrand quantity $\left(\Sigma_{11}^{\infty}-\Sigma_{11}(l)\right)$ as a function of distance from the crack between $l=0$ and $l=+\infty$ on the other hand. These two values are then compared. Moreover, as the values of the energy release rate of a small crack aligned with the principal stretch directions $\left(\theta=0^{\circ}\right.$ and $\left.\theta=90^{\circ}\right)$ are known under uniaxial extension, pure shear and equibiaxial extension [26], it is wise to begin the investigation with these simple cases. The verification will continue afterwards by considering the same three previous far-field loading cases but with non-trivial crack orientations $(\theta \in] 0^{\circ}, 90^{\circ}[)$.

Tables 1 and 2 provide the energy release rate of a small crack under uniaxial tension $(B=-0.5)$ for various crack orientations for "small" strain (stretch ratio equal to 1.1) and large strain (stretch ratio equal to 2.5 ), respectively. The relative discrepancy between our method and the $J$-integral computed by Abaqus never exceeds $4 \%$. Given that the improper integral is estimated on a bounded domain, this discrepancy turns out to be very satisfactory.

Similar computations have been carried out in the cases of a small crack under pure shear $(B=0)$ and equibiaxial tension $(B=-1)$ and for the same values of far-field parameters $\theta$ and $\lambda$ and for $B=0$. Once again, the relative discrepancy remains always smaller than $4 \%$.

All these numerical results prove and validate the theoretical expression Eq. (13) of the energy release rate of a small crack under arbitrary multiaxial loading.

\subsubsection{The energy release rate is proportional to crack length}

The general nature of the proportionality relationship Eq. (19) needs to be numerically verified. Indeed in the case where the crack is not aligned with one of the principal stretch directions, this result is not so obvious. Remembering the derivation carried out in [2], we wonder whether the dimensional considerations made there remain valid when the crack is not perpendicular to the maximum principal stretch ratio direction.

Our validation consists in considering two different sizes for small crack $c_{1}$ and $c_{2}$ under the same far-field loading conditions

Table 1

Energy release rate $\left(\mathrm{kJ} / \mathrm{mm}^{2}\right)$ of a small crack of length $c=10 \mathrm{~mm}$ under uniaxial tension $(\lambda=1.1)$ for various crack orientations.

\begin{tabular}{lll}
\hline Uniaxial tension $(B=-0.5)$ and $\lambda=1.1$ & \\
\hline Crack orientation $\theta\left(^{\circ}\right)$ & $J$ by Abaqus & $2 \int_{0}^{+\infty}\left(\Sigma_{11}^{\infty}-\Sigma_{11}(l)\right) d l$ \\
\hline 0 & 0.81891 & 0.80973 \\
15 & 0.7525 & 0.73390 \\
30 & 0.60428 & 0.59712 \\
45 & 0.40137 & 0.40454 \\
60 & 0.19935 & 0.20596 \\
75 & 0.05306 & 0.05583 \\
90 & 0.00000 & 0.00000 \\
\hline
\end{tabular}

Table 2

Energy release rate $\left(\mathrm{kJ} / \mathrm{mm}^{2}\right)$ of a small crack of length $c=10 \mathrm{~mm}$ under uniaxial tension $(\lambda=2.5)$ for various crack orientations.

\begin{tabular}{lcc}
\hline \multicolumn{2}{l}{ Uniaxial tension $(B=-0.5)$ and $\lambda=2.5$} \\
\hline Crack orientation $\theta\left(^{\circ}\right)$ & $J$ by Abaqus & $2 \int_{0}^{+\infty}\left(\Sigma_{11}^{\infty}-\Sigma_{11}(l)\right) d l$ \\
\hline 0 & 78.7453 & 75.97629 \\
15 & 73.4208 & 70.89085 \\
30 & 59.05740 & 57.04331 \\
45 & 39.41430 & 38.11648 \\
60 & 19.76130 & 19.18989 \\
90 & 5.30489 & 5.19568 \\
\hline 0
\end{tabular}


and then in comparing the ratio with their respective energy release rate for the widest possible range of far-field parameters $\lambda, B$ and $\theta$. For the first crack length, we reuse the results of the previous section $\left(c_{1}=10 \mathrm{~mm}\right)$; and the second crack is chosen to be half the size of $c_{1}: c_{2}=c_{1} / 2=5 \mathrm{~mm}$. The finite element models used for each crack size admit exactly the same features (see above) except for the arrangement of mesh elements. Indeed, in order to improve the accuracy of the comparison between the two cases, it is judicious to mesh the vicinity of the two small cracks with meshes that are related by a scale factor equal to the ratio between these two crack lengths. The near-crack geometry was discretized with a very regular mesh (right-angled quadrilaterals) that permits to control the elements sizes along with their distribution throughout the mesh. Thus, at the scale of the crack, the mesh density in the vicinity of the crack was the same for both finite element models.

All the results obtained demonstrate that, for $B$ increasing from -0.5 to 1 by increments of 0.25 , for $\lambda$ increasing from 1.1 to 5.0 by increments of 0.1 , and for $\theta$ increasing from $0^{\circ}$ to $90^{\circ}$ by

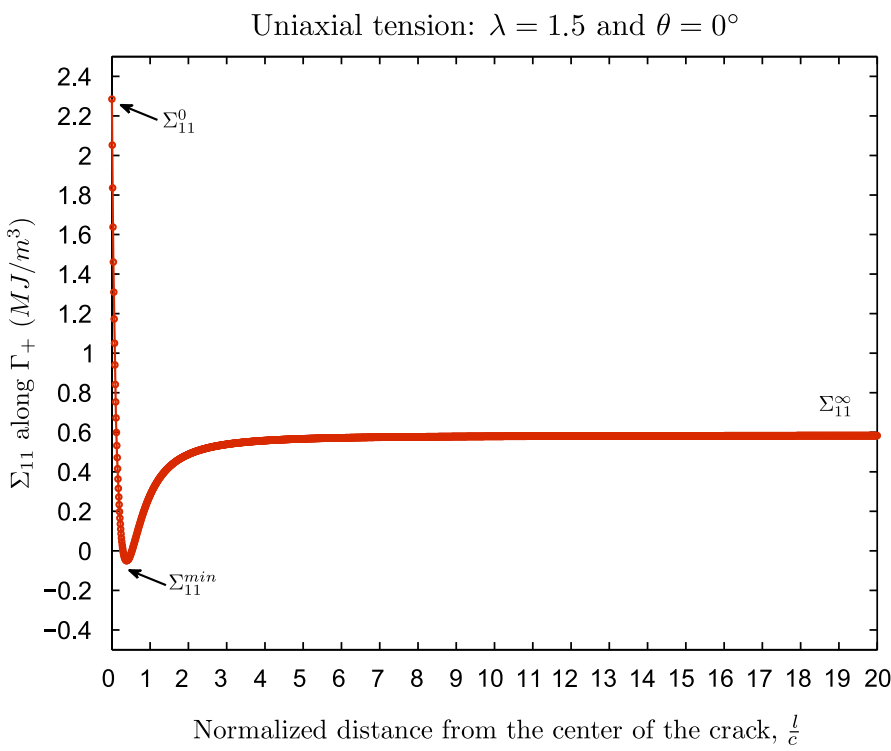

increments of $15^{\circ}$ we always verify

$\frac{J\left(B, \lambda, \theta, c_{1}=10 \mathrm{~mm}\right)}{J\left(B, \lambda, \theta, c_{2}=5 \mathrm{~mm}\right)}=2$.

Considering these numerical results, we are henceforth convinced that the energy release rate of a small crack is proportional to the crack length whatever the far-field in which the small crack is embedded. In other words, the proportionality relationship is proven valid even when the crack is not aligned with one of the principal stretch directions.

\subsection{Analysis of the configurational stress imbalance}

On referring now to the mechanical interpretation of Eq. (13) stated above, we know that the $J$-integral of a small crack corresponds to the imbalance between the uniform configurational stresses acting on the vertical right-hand side boundary $\Gamma_{B}$ and the varying configurational stresses acting on the vertical left-hand side boundary $\Gamma_{+}$of the contour $\Gamma$ (see Fig. 1). We now attempt to

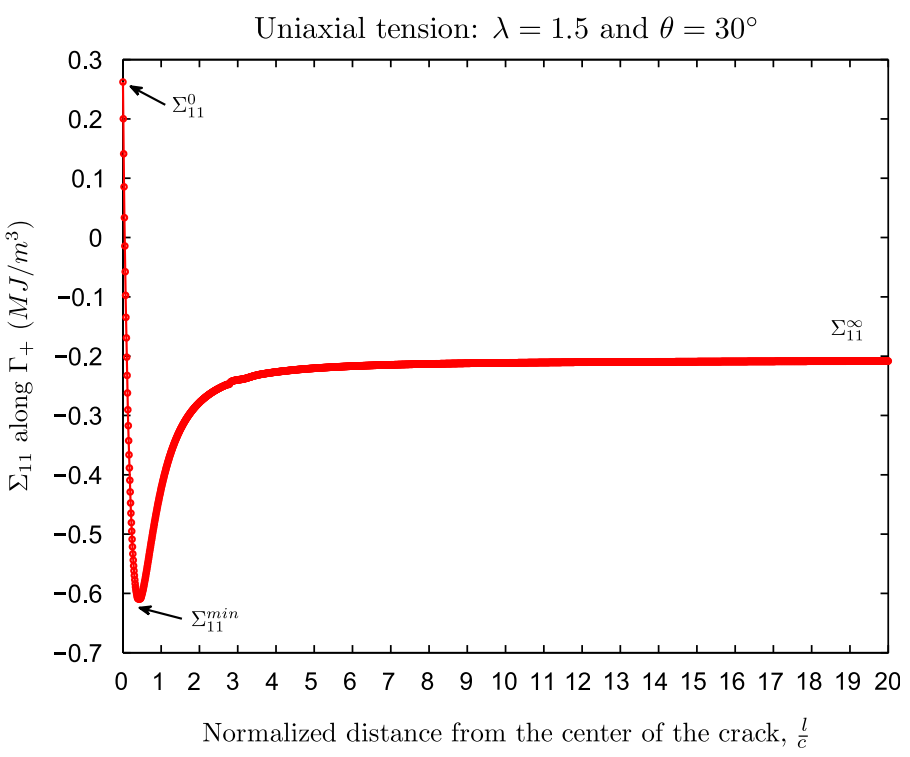

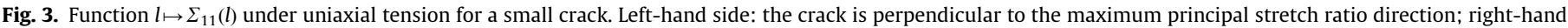
side: the crack that is slanted with respect to the principal stretch ratio directions.
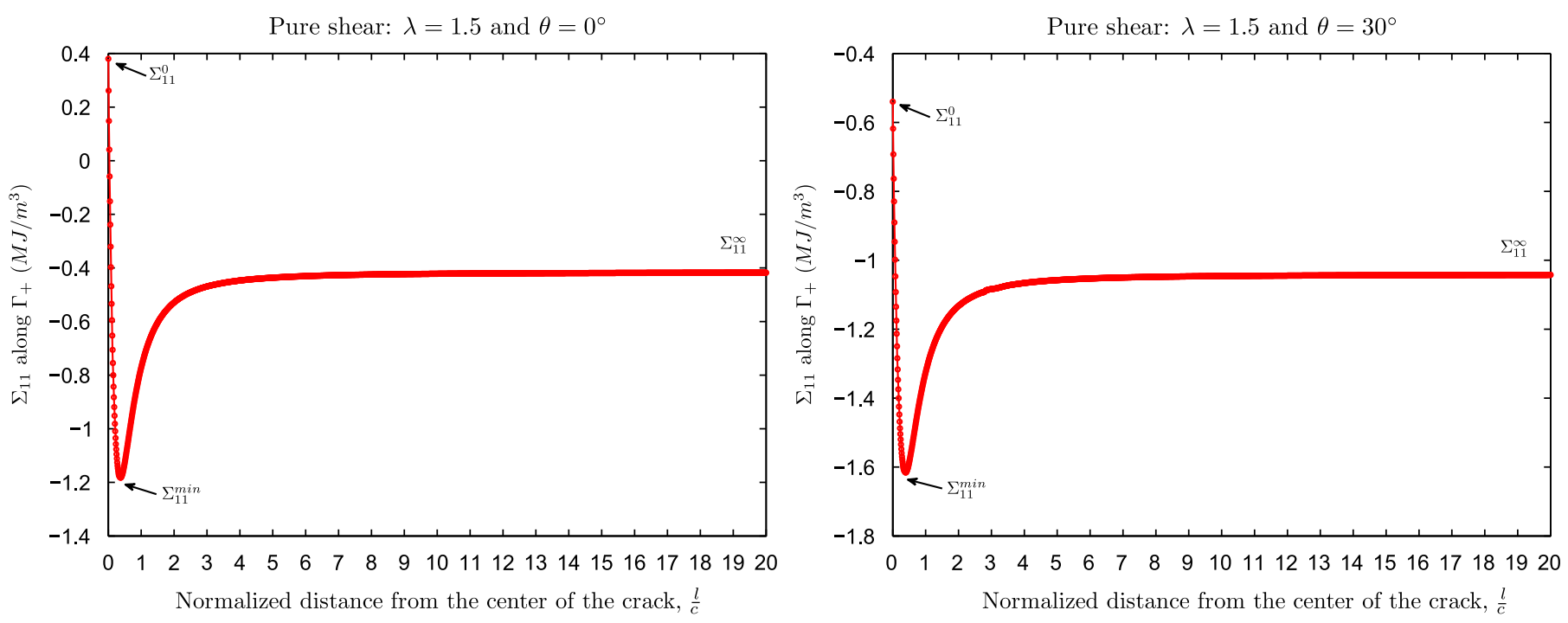

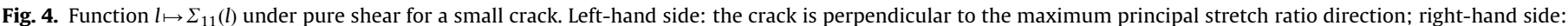
the crack that is slanted with respect to the principal stretch ratio directions. 


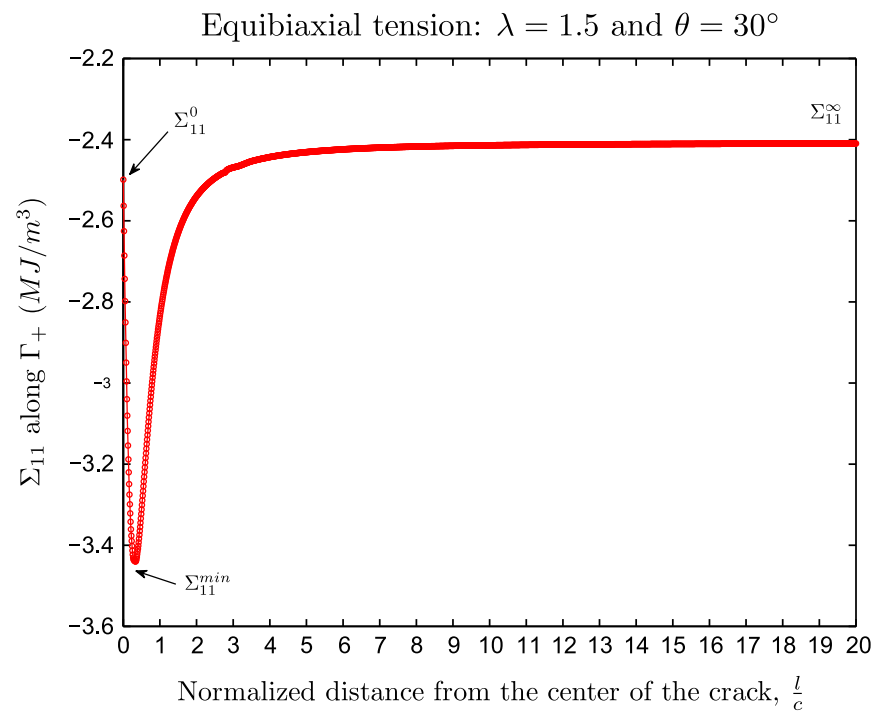

Fig. 5. Function $l \mapsto \Sigma_{11}(l)$ under equibiaxial tension for a small crack. This result is the same for all crack orientations.

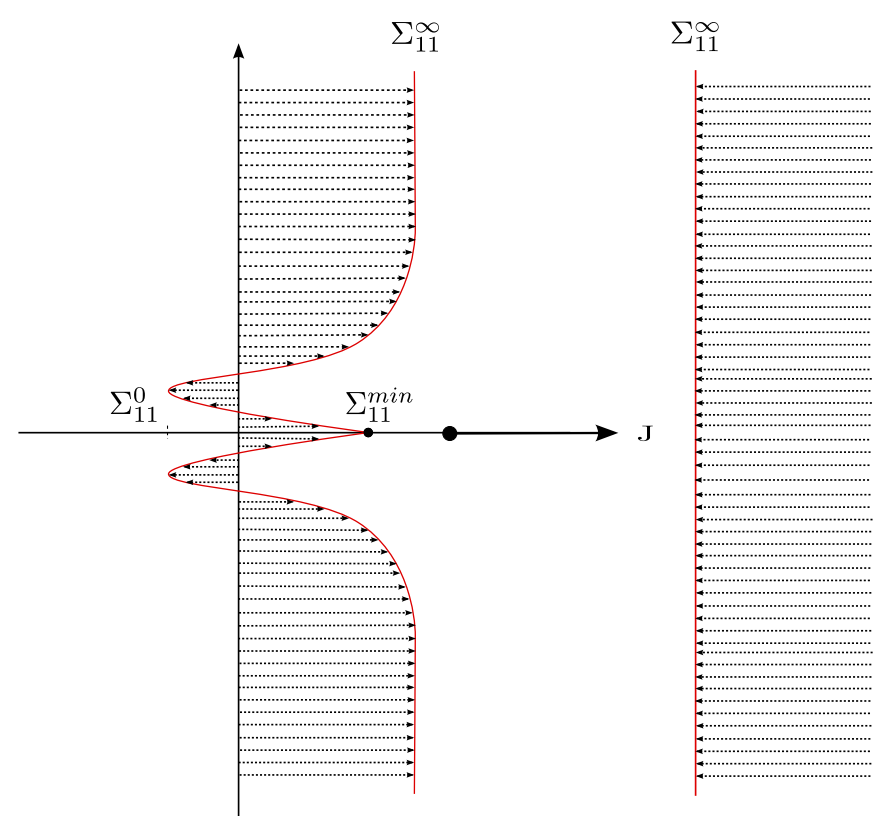

Fig. 6. Configurational force balance.

make an account of how the near-crack variations of the function $l \mapsto \Sigma_{11}(l)$ make up for the energy release rate of a small crack. After computing expression Eq. (13) under uniaxial tension, pure shear and equibiaxial tension for various crack orientations and different stretch ratios, we noticed a remarkable feature in the plot of $l \mapsto \Sigma_{11}(l)$ : all these plots have the same aspect. Indeed, it turns out that whatever the values of the far-field parameters $\lambda, B$ and $\theta$, this function always first decreases and then increases up to the farfield value with a minimum that occurs at $l \approx c / 2$. The position of this minimum approaches $l=0$ as the stretch ratio increases. Figs. 3-5 concretely illustrate this property by providing examples of plots of $l \mapsto \Sigma_{11}(l)$ for different values of biaxiality $B$ (uniaxial tension $B=-0.5$, pure shear $B=0$ and equibiaxial tension $B=1$ ) and crack orientation $\theta$ at a stretch ratio $\lambda=1.5$.

All these plots highlight three characteristic values:

- $\Sigma_{11}^{0}: \Sigma_{11}(l=0)$, i.e. the value of $\Sigma_{11}$ at the intersection of $\Gamma_{+}$ with the crack face,
- $\Sigma_{11}^{\min }: \min _{l \in[0, \infty[}\left\{\Sigma_{11}(l)\right\}$, i.e. the minimum value of $\Sigma_{11}$ reached at a distance from the center of the crack on the order of $c / 2$,

- $\Sigma_{11}^{\infty}: \lim _{l \rightarrow \infty} \Sigma_{11}(l)$, i.e. the asymptotic value of $\Sigma_{11}$, which is actually equal to that in the crack-free problem (far-field).

By gradually refining the mesh of the finite element model, we have verified that the values of $\Sigma_{11}^{0}$ and $\Sigma_{11}^{\min }$ always converge, i.e. they are both independent from the mesh. Thus, it permits to conclude that, in addition to $\Sigma_{11}^{\infty}, \Sigma_{11}^{\min }$ and $\Sigma_{11}^{0}$ depend on the farfield loading conditions only; in other words, they only depend on the far-field parameters $\lambda, B$ and $\theta$. Actually this result was predictable because singular values (infinite values) of the mechanical fields take place only at crack tip. Therefore, at any point along $\Gamma_{+}$, all the mechanical fields are regular and it is legitimate to claim that the features noticed in the plots of $l \mapsto \Sigma_{11}(l)$ are general properties that intrinsically characterize a small crack whatever the principal stretch ratios $\lambda$ and $\lambda^{B}$ of the multiaxial far-field in which the crack is embedded and whatever its orientation $\theta$ with respect to the principal stretch ratio directions.

Fig. 6 illustrates the balance of configurational stresses acting on a small crack. As established in Eq. (13), we only need to consider the configurational stress component associated with the crack growth direction. In the figure, the projection onto the crack plane of the configurational force vector $\mathbf{J}$ acting at crack tip, with magnitude equal to the energy release rate of the crack, is obtained by simply summing the configurational stress that act on the leftand right-hand side cutting planes: (i) a uniform configurational stress acts on the right-hand side plane, located far from the crack; and (ii) the left-hand side plane, which cuts the center of the crack, is acted on by a distribution of configurational stress $\left(l \mapsto \Sigma_{11}(l)\right)$, which admits an asymptote to the far-field value and varies in the near-field due to the effects of crack face unloading.

\section{Conclusions}

The energy release rate of a small crack can be computed by considering the balance of configurational stress acting on two planes: one cutting the center of the crack face, and another at an infinite distance in front of the crack tip. The computation can be made for arbitrary multiaxial loading conditions, and it provides a means of bridging between continuum mechanics, through farfield quantities, and fracture mechanic, through the energy release rate, views of crack evolution in hyperelastic solids; it is particularly suited to the requirements of critical plane analysis classically adopted in fatigue problems.

The relevance of our approach was demonstrated through its ability to frame and prove the widely held view that the energy release rate of a small crack is proportional to the size of the crack, irrespective of the state of loading or the orientation of the crack.

There is a significant difference between the near-field configurational stress and the far-field value. This difference is associated with unloading of the crack face, and it is balanced by the concentrated material force at crack tip, which magnitude is equal to the energy release rate of the crack.

We have plotted the configurational stress balance for the benchmark cases of uniaxial tension, pure shear and equibiaxial tension, and for cracks inclined with respect to the axis of loading. First, energy release rates compared well with previously known results. Second, in all cases, the configurational stress balance exhibits the same characteristic features: an asymptote to the farfield value, a non-zero value at crack face, and a locally minimum value at some distance $\approx c / 2$ from the crack face. 


\section{Acknowledgments}

The authors are grateful for financial support by the Centre Technique des Industries Mécaniques (CETIM, Technocampus EMC2, ZI du Chaffault, 44340 Bouguenais, France), and especially Dr. P. Castaing.

\section{References}

[1] A. Griffith, The phenomena of rupture and flow in solids, Philosophical Transactions of the Royal Society of London A 221 (1921) 163-198.

[2] R.S. Rivlin, A.G. Thomas, Rupture of rubber. I. Characteristic energy for tearing, Journal of Polymer Science 10 (1953) 291-318.

[3] J.F. Roach, Crack Growth in Elastomers Under Biaxial Stresses, Ph.D. Thesis, University of Akron, USA, 1982

[4] W.V. Mars, Cracking energy density as a predictor of fatigue life under multiaxial conditions, Rubber Chemistry and Technology 75 (2002) 1-17.

[5] N. Saintier, N. André, G. Cailletaud, R. Piques, Crack nucleation and propagation under multiaxial fatigue in natural rubber, International Journal of Fatigue 28 (2006) 61-72.

[6] A. Andriyana, E. Verron, Prediction of fatigue life improvement in natural rubber using configurational stress, International Journal of Solids and Structures 44 (2007) 2079-2092.

[7] E. Verron, A. Andriyana, Definition of a new predictor for multiaxial fatigue crack nucleation in rubber, Journal of the Mechanics and Physics of Solids 56 (2008) 417-443.

[8] J.-B. Brunac, O. Gérardin, J.-B. Leblond, On the heuristic extension of Haigh's diagram for the fatigue of elastomers to arbitrary loadings, International Journal of Fatigue 31 (2009) 859-867.

[9] A. Andriyana, N. Saintier, E. Verron, Configurational mechanics and critical plane approach: concept and application to fatigue failure analysis of rubberlike materials, International Journal of Fatigue 32 (2010) 1627-1638.

[10] G.A. Maugin, Material Inhomogeneities in Elasticity, Chapman and Hall, London, 1993.

[11] G.A. Maugin, Material forces: concepts and applications, Applied Mechanics Reviews 48 (1995) 213-245.

[12] C. Truesdell, W. Noll, Handbuch der Physik Bd. III/3, Vol. The Nonlinear Field Theories of Mechanics, Springer-Verlag, Berlin, 1965.
[13] P. Steinmann, D. Ackermann, F.J. Barth, Application of material forces to hyperelastostatic fracture mechanics. II. Computational setting, International Journal of Solids and Structures 38 (2001) 5509-5526.

[14] E. Verron, Study of the simple extension tear test sample for rubber with configurational mechanics, International Journal of Fracture 147 (1-4) (2007) 219-225.

[15] M. Kaliske, B. Näser, C. Meiners, Inelastic fracture mechanics for tire durability simulations, Tire Science and Technology 35 (3) (2007) 239-250.

[16] E. Verron, Configurational mechanics: a tool to investigate fracture and fatigue of rubber, Rubber Chemistry and Technology 83 (2010) 270-281.

[17] D. Parks, The virtual crack extension method for nonlinear material behavior Computer Methods in Applied Mechanics and Engineering 12 (1977) 353-364.

[18] C. Shih, B. Moran, T. Nakamura, Energy release rate along a three-dimensional crack front in a thermally stressed body, International Journal of Fracture 30 (1986) 79-102.

[19] K.N. Shivakumar, P.W. Tan, J.C. Newman, A virtual crack-closure technique for calculation stress intensity factors for cracked three-dimensional bodies, International Journal of Fracture 36 (1988) 7371-7971.

[20] P. Steinmann, Application of material forces to hyperelastostatic fracture mechanics. I. Continuum mechanical setting, International Journal of Solids and Structures 37 (2000) 7371-7391.

[21] R. Mueller, G.A. Maugin, On material forces and finite element discretizations, Computational Mechanics 29 (2002) 52-60.

[22] J.R. Rice, A path independent integral and the approximate analysis of strain concentration by notches and cracks, Journal of Applied Mechanics 35 (1968) 379-386.

[23] J.D. Eshelby, The force on an elastic singularity, Philosophical Transactions of the Royal Society of London A 244 (1951) 87-112.

[24] J.D. Eshelby, The elastic energy-momentum tensor, Journal of Elasticity 5 (3-4) (1975) 321-335.

[25] P. Chadwick, Applications of an energy-momentum tensor in non-linear elastostatics, Journal of Elasticity 5 (3-4) (1975) 249-258.

[26] O.H. Yeoh, Relation between crack surface displacements and strain energy release rate in thin rubber sheets, Mechanics of Materials 34 (8) (2002) 459-478.

[27] M. Aït-Bachir, Prediction of Crack Initiation in Elastomers in the Framework of Configurational Mechanics (in French), Ph.D. Thesis, Ecole Centrale de Nantes, Nantes (France), 2010. 